\title{
Personality types in individuals with type 1 and type 2 diabetes
}

\author{
A Rouland ${ }^{1}$, J-C Chauvet-Gelinier ${ }^{2,3}$, A-L Sberna ${ }^{1,3}$, E Crevisy ${ }^{1}$, P Buffier ${ }^{1}$, T Mouillot ${ }^{1}$, J-M Petit ${ }^{1,3}$ and B Vergès ${ }^{1,3}$ \\ ${ }^{1}$ Endocrinology Diabetics and Metabolic Disorders Department, Dijon University Hospital, Dijon, France \\ ${ }^{2}$ Psychiatry Unit, Department of Neurosciences, Dijon University Hospital, Dijon, France \\ 3INSERM Unit, LNC-UMR 1231, University of Burgundy, Dijon, France
}

Correspondence should be addressed to A Rouland: alexia.rouland@chu-dijon.fr

\begin{abstract}
Objective: The Type A personality, characterized by impatience, strong career ambition and competitiveness, is associated with greater sensitivity to external stress. Type 1 diabetes (T1D) is an auto-immune disease, which is potentially influenced by stress, unlike type 2 diabetes (T2D). The aim of this study was to assess whether individuals with T1D and T2D exhibited significant differences on the Type A personality scale. We also assessed personality in patients with thyroid auto-immune diseases to validate potential links between auto-immune disease and Type A personality.

Design and methods: The Bortner questionnaire was used to assess Type A personality in 188 patients with T1D, 430 patients with T2D and 85 patients with auto-immune thyroid disease (Graves' disease or Hashimoto's thyroiditis).

Results: Type A Bortner scores were significantly higher in T1D patients than in T2D patients (188 \pm 34 vs $177 \pm 36, P<0.0001)$. Patients with auto-immune thyroid diseases and T1D patients had similar Type A Bortner scores ( $189 \pm 33$ vs $188 \pm 34, P=0.860$ ). Conclusion: Patients with auto-immune T1D have higher Type A scores than T2D patients. Furthermore, patients with auto-immune thyroid disease also have elevated Type A scores similar to those observed in type 1 diabetes, suggesting that an elevated Type A score in T1D is potentially related to its auto-immune origin. This suggests a possible link between Type A personality and auto-immune diseases via stress-triggering psychobiological pathways. The different personality score between T1D and T2D is an important factor, which could influence self-care coping strategies in diabetes and long-term prognosis.
\end{abstract}

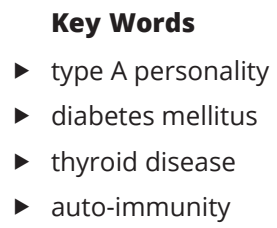

\section{Introduction}

Type 1 (T1D) and type 2 diabetes (T2D) are chronic diseases in which psychological determinants are an important field of research, not only to improve personalized treatment and educational strategies, but also to directly influence health outcomes. Most published scientific reports to date have focussed on anxiety and depressive disorders as the two major detrimental co-morbid conditions of T1D and T2D, but little is known about the nature and influence of stable personality traits. Type A personality is a psycho-behavioral profile that has been studied for many years and which has been associated with various health issues (e.g. cardiovascular diseases and diabetes) $(1,2,3,4,5,6)$. Type A personality is characterized by impatience, a need for personal fulfillment, career ambition and competitive drive. In addition, when faced with adversity, Type A personality is associated with problem-focused coping strategies (7) in a very close way, together with conscientiousness (8), which is a personality dimension characterized by control, organization and assiduousness. Indeed, Type A personality was previously linked to conscientiousness (8) in terms of the 'goalstriving' dimension, as a psychological ability to regulate

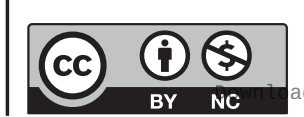


cognitions and behaviors toward identified goals. Several reports showed that Type A has been associated with more problem-focused coping strategies (7), including the search for appropriate medical monitoring (9) and a greater sense of self-efficacy (10).

In terms of health behaviors, Type A has been linked to increased interest in finding appropriate medical treatment (11) and adherence to disease screening $(9,12$, $13,14)$.

Although early reports stated that Type A was associated with a greater risk of developing coronary heart disease, subsequent studies have dismissed this link and shown that Type A personality may actually have a positive impact on health, likely due to the need for personal fulfillment and the competitive spirit $(1,2,15$, 16). Indeed, in T1D, Type A personality has been associated with lower cardiovascular and all-cause mortality (15) and, in both T1D and T2D, Type A personality has been shown to be independently associated with reduced plasma C-reactive protein levels (1). Thus, Type A personality appears to be a psychological factor worth considering in clinical practice. However, little is known about the manifestation of Type A personality in T1D and T2D, which are dissimilar diseases in many ways. Type A personality has been studied in patients with diabetes, but because T1D and T2D were mixed together in the same analysis group, it was not possible to tease out differences between T1D and T2D (17). Some studies have been performed on Type A personality and T1D (18), but until now, a comparison of Type A personality scores has never been done for T1D and T2D.

It seems worthy of investigation because the pathophysiology of the two disorders differs considerably. One major difference is that T1D is an auto-immune disease whereas T2D is not. Many data indicate that stress could be a trigger factor for the onset of auto-immune diseases, and several studies suggest that stress is not only a participating factor, but can, in fact, cause disease and worsen a patient's condition $(19,20,21,22)$.

Because Type A people are particularly reactive to stress (23), they may be more prone to auto-immune diseases. Therefore, we hypothesized that patients with T1D would be prone to higher Type A stress-sensitive personality levels than patients with T2D.

This prompted us to evaluate Type A personality in a large population of patients with diabetes, including 188 patients with T1D and 430 patients with T2D, a nonauto-immune disease.

We planned to first assess T1D and T2D patients on a Type A personality scale and to compare the higher susceptability to Type A behavior patterns. Then, if a statistically significant difference was observed, we would carry out an additional assessment and compare the Type A profiles of patients with T1D and patients presenting with other auto-immune disease (Graves' disease or Hashimoto's thyroiditis), in order to validate the hypothesis that increased Type A personality traits is related to the auto-immune profile of the patients suffering from or who develop T1D.

\section{Materials and methods}

\section{Patients with diabetes}

We prospectively included 618 patients (in- and outpatients) from the department of Endocrinology, Diabetes, and Metabolic Diseases of the University Hospital of Dijon over a 43-month period. All the patients presenting with T1D or T2D, Hashimoto's or Graves' disease were included.

All the patients were older than 18 years. Auto-immune T1D was confirmed with samples testing positively for diabetes-specific antibodies (anti-GAD, anti-IA2 and antiislets). Patients with type 2 diabetes did not suffer from any known auto-immune disease.

Since the tests were written in French, anyone who was not fluent in the French language was excluded in order to guarantee a reliable measurement on the various scales for each patient. For the same reason, we excluded illiterate patients or patients suffering from memory or psychiatric disorders that would make reliable collection of the test results impossible.

\section{Patients with auto-immune thyroid diseases}

Eighty-five patients with auto-immune thyroid disease (Graves' disease or Hashimoto's thyroiditis) were included. These patients were out-patients followed by the physicians in the department of Endocrinology, Diabetes, and Metabolic Diseases of the Dijon University Hospital or in the Endocrinology Department of two other hospitals in the region.

Auto-immune thyroid disease was confirmed in each patient following testing for disease specific antibodies (TSH-receptor antibody for Graves' disease and thyroid peroxidase (TPO) antibodies for Hashimoto's thyroiditis). None of the patients had diabetes. Patients with autoimmune thyroid disease were asked to complete the same questionnaires as patients with diabetes.

In the present study, we intentionally decided not to include patients with severe auto-immune disease, 
such as lupus or rheumatoid arthritis, where coping strategies and treatments do not necessarily result in pain relief or good outcomes. Our intention was ensure that the severity of the disease did not influence the results of the psychological tests.

\section{Psychological tests}

Overall, three tests were distributed to patients who then completed the questionnaires themselves, alone, in a quiet place. The tests measured Type A personality (Bortner scale) and for any potentially associated psychological confounders such as anxiety and depression. Hostility was not measured, as the Bortner scale is not designed for it.

\section{Bortner scale (Type A personality)}

Type A personality was evaluated using the Bortner rating scale (24). We used the French version of the Bortner scale, which has been validated in the French language $(25,26)$. The test is made up of 14 items, each consisting of two statements at opposite ends of a spectrum (e.g. 'never late' and 'do not pay attention to being late for appointments'). The patient places themselves on each scale according to their own personality traits. The score is calculated for each item by counting the number of ticks separating one end of the spectrum and the point placed by the patient. For each item, the maximum score is 24 and the minimum score is 0 . The total score can thus be used as a continuous variable with higher scores indicating Type A personality.

\section{Zigmund and Snaith test (anxiety and depression)}

The French version of the Zigmund and Snaith test (hospital anxiety and depression scale) was used to assess the symptoms of anxiety and depression (27). This test has 14 items: seven items for anxiety and seven items for depression. There are four choices for each item and the patient must select the one that fits them best (e.g. 'I feel tense or irritated: most of the time, often, sometimes, never').There is a score of 0 to 3 points per item depending on the answer selected by the respondent. The points for each section are then added up: the closer the total is to 21 , the greater the anxiety or depression score.

\section{Statistical analyses}

Descriptive data are analyzed as mean \pm s.D. The score obtained on the Bortner scale was considered continuous data.
The Student's $t$-test was used to compare quantitative variables and the chi-square test to compare qualitative variables. The comparison of means between three groups of patients was performed by ANOVA and post-hoc tests (by least significance difference) were used to compare each mean with another one separately. The comparison of quantitative variables between two groups after adjustment for a quantitative variable was performed by analysis of covariance (ANCOVA). SPSS software was used for all statistics. A two-tailed probability of $P<0.05$ was accepted as statistically significant.

\section{Ethics}

The study was approved by the ethical committee of the Dijon University Hospital (CPP-Est I). Prior to the interviews and assessments, the interviewer described the aim of the study to each participant and obtained their full verbal consent. The study was carried out in accordance with the recommendations of the local ethics committee, which approved the oral consent on the basis that the research would not cause serious harm to the study participants (NCT01961466).

\section{Results}

\section{Patients with T1D vs T2D}

\section{Patients characteristics}

The characteristics of the patients with T1D and T2D are shown in Table 1. The sex ratio was similar for T1D and T2D patients. Patients with T2D were older than patients with T1D (Table 1).

We included 188 patients with T1D and 430 patients with T2D.

There were 91 women and 97 men, according to Table 1, in the T1D group, and the average age was 39.9 years.

The T2D group was $43.4 \%$ women (or 190 patients). The average age was 60.5 years.

\section{Comparison of Bortner, anxiety and depression scores in patients with T1D and T2D}

Table 2 shows the Bortner Type A, anxiety and depression scores in patients with T1D and T2D. Type A scores were significantly higher in patients with T1D than in patients with T2D $(188 \pm 34$ vs $177 \pm 36, P<0.0001)$. Patients with T2D had a significantly higher depression score than T1D patients $(6.09 \pm 3.41$ vs $5.02 \pm 3.46)$. The Type A

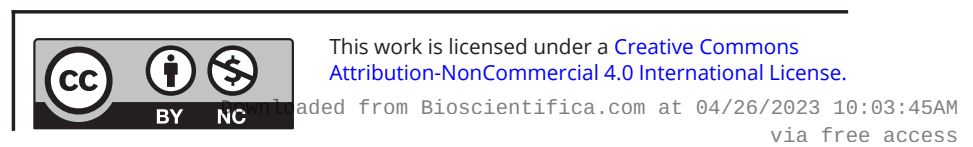


Table 1 General characteristics of type 1 and type 2 diabetic patients (mean \pm s.D.).

\begin{tabular}{l}
\hline \\
\hline Age (years) \\
Sex ratio (women), $n$ (\%) \\
$\mathrm{BMI}\left(\mathrm{kg} / \mathrm{m}^{2}\right)$ \\
$\mathrm{HbA} 1 \mathrm{c}(\mathrm{mmol} / \mathrm{mol})(\%)$ \\
$\mathrm{LDL}-\mathrm{cholesterol}(\mathrm{mmol} / \mathrm{L})$ \\
$\mathrm{HDL}$-cholesterol $(\mathrm{mmol} / \mathrm{L})$ \\
Triglycerides $(\mathrm{mmol} / \mathrm{L})$ \\
Systolic BP $(\mathrm{mmHg})$ \\
Diastolic BP $(\mathrm{mmHg})$ \\
\hline
\end{tabular}

\begin{tabular}{c}
\hline Type 1 diabetes \\
\hline$n=188$ \\
\hline $39.9 \pm 14.3$ \\
$91(48.4)$ \\
$25.0 \pm 6.3$ \\
$93.9(8.6) \pm 19.7(1.8)$ \\
$2.76 \pm 0.80$ \\
$1.54 \pm 0.44$ \\
$1.10 \pm 0.50$ \\
$129 \pm 18$ \\
$74 \pm 10$
\end{tabular}

score remained significantly higher in patients with T1D compared to patients with T2D after adjustment for age $(P<0.0001)$ and for depression $(P<0.0001)$.

\section{Patients with T1D vs auto-immune thyroid disease}

\section{Patients characteristics}

Because patients with T1D featured significantly higher Bortner Type A scores, we aimed to compare their test results with the results of patients with thyroid autoimmune diseases. Our objective was to see whether the augmented Type A score observed in T1D was potentially linked to the auto-immune profile of T1D.

Among the 85 patients with thyroid auto-immune diseases, 51 had Graves' disease and 34 had Hashimoto's thyroiditis. The main patient characteristics, Bortner Type A score, and anxiety and depression scores of the patients with thyroid auto-immune diseases and with T1D are shown in Tables 3 and 4. Patients with T1D were younger than patients with auto-immune thyroid diseases $(39.9 \pm 14.3$ vs $48.9 \pm 14.8, P<0.0001)$. There was a larger proportion of women in the auto-immune thyroid diseases group than in the T1D group (91 women vs 77 women, $P<0.0001)$.

Table 2 Comparison of diabetic patients groups in terms of age, sex ratio, Bortner score, anxiety and depression. Data are expressed as mean \pm S.D.

\begin{tabular}{|c|c|c|c|}
\hline & $\begin{array}{c}\text { Type } 1 \\
\text { diabetes }\end{array}$ & $\begin{array}{c}\text { Type } 2 \\
\text { diabetes }\end{array}$ & $\mathbf{P}$ \\
\hline Age (years) & $39.9 \pm 14.3$ & $60.5 \pm 10.6$ & $<0.000$ \\
\hline Sex ratio (M/W) & $91 / 97$ & $240 / 190$ & NS \\
\hline Bortner ( 0 to 336 ) & $188 \pm 34$ & $177 \pm 36$ & $<0.000$ \\
\hline Anxiety (0 to 21$)$ & $9.78 \pm 4.27$ & $9.46 \pm 3.91$ & NS \\
\hline $\begin{array}{l}\text { Depression } \\
\text { (0 to } 21)\end{array}$ & $5.02 \pm 3.46$ & $6.09 \pm 3.41$ & 0.001 \\
\hline
\end{tabular}

\begin{tabular}{ccc}
\hline Type 2 diabetes & & $\boldsymbol{P}$ \\
\hline$n=430$ & $<0.0001$ \\
$60.5 \pm 10.6$ & $\mathrm{NS}$ \\
$190(43.4)$ & $<0.0001$ \\
$30.9 \pm 8.4$ & $\mathrm{NS}$ \\
$91.8(8.4) \pm 19.7(1.8)$ & 0.005 \\
$2.47 \pm 1.06$ & $<0.0001$ \\
$1.16 \pm 0.46$ & $<0.0001$ \\
$2.12 \pm 1.47$ & 0.001 \\
$135 \pm 18$ & 0.008 \\
$77 \pm 11$ &
\end{tabular}

Comparison of Bortner scores in patients with T1D and auto-immune thyroid disease

Patients with auto-immune thyroid diseases and patients with T1D had similar Bortner Type A scores (189 \pm 33 vs $188 \pm 34, P=0.860$ ) (Table 4).

The mean Bortner Type A score remained statistically similar in both patient groups after adjustment for age $(P=0.86)$ or for sex ratio $(P=0.84)$.

\section{Comparison of Bortner scores in patients with T1D, T2D and auto-immune thyroid disease}

ANOVA showed a statistically significant difference between the three groups of patients (T1D patients, T2D patients and auto-immune thyroid disease patients) $(P<0.0001, \mathrm{~F}=8.81)$.

Then, we compared the mean of each group with the mean of another one using post-hoc tests. We found that the mean Bortner score was significantly higher in T1D patients than in T2D patients $(P<0.0001)$, that the mean Bortner score was significantly higher in patients with auto-immune thyroid disease than in T2D patients $(P=0.004)$, and that the mean Bortner score in T1D patients was not different from the Bortner score in patients with auto-immune thyroid disease $(P=0.86)$.

Moreover, we compared the Type A Bortner score between patients with auto-immune disease (T1D and auto-immune thyroid disease) and with non-auto-immune disease (T2D). The difference was, once again, statistically significant. The Type A Bortner score in patients with auto-immune diseases was $188 \pm 33$ and in patients with no auto-immune disease was $177 \pm 36(P<0.0001)$.

Figure 1 shows the Bortner Type A scores of patients with T1D, auto-immune thyroid diseases and patients with T2D. We clearly see that auto-immune T1D and auto-immune thyroid disease patients had similar Bortner scores, which were significantly higher than the scores found in the T2D group $(P<0.0001)$.

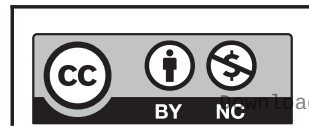


Table 3 Characteristics of patients with auto-immune thyroid disease and type 1 diabetes.

\begin{tabular}{l} 
\\
\hline Age (years) \\
Sex ratio (women), $n(\%)$ \\
Auto-immune diseases: \\
Graves' disease, $n(\%)$ \\
Hashimoto's thyroiditis, $n(\%)$ \\
\hline
\end{tabular}

\begin{tabular}{c}
\hline Auto-immune thyroid diseases \\
\hline$n=88$ \\
\hline $48.29 \pm 14.761$ \\
75 \\
49 \\
33
\end{tabular}

\begin{tabular}{ccc}
\hline Type 1 diabetes & & $\boldsymbol{P}$ \\
\hline$n=188$ & & $<0.0001$ \\
$39.46 \pm 14.106$ & & \\
$92(48.9)$ & & \\
0 & \\
0 &
\end{tabular}

\section{Discussion}

We present here the first comparative analysis of Type A personality in individuals with T1D and T2D. We demonstrate that patients with auto-immune T1D have higher Type A scores than patients with T2D, indicating a more pronounced Type A profile in T1D. Furthermore, we show that patients with auto-immune thyroid disease also have an elevated Bortner Type A score similar to what is observed in T1D. This suggests that elevated Type A score in T1D is likely linked to its auto-immune origin.

In the present study, the Type A score of patients with T2D was similar to the score reported in the general population. Indeed, various studies indicated that the Bortner score in the general population is around 175: Bucik et al. found an average Bortner score of 175 in 743 people (28) and another study in 2012 found an average score of 167 in 50 healthy subjects (29). In the present study, however, the mean Type A score for the T1D group (188) was significantly higher than the mean Type A score of the general population, suggesting that T1D patients have a more pronounced Type A profile than both T2D patients and the general population.

The reasons for the increased Type A personality score in patients with T1D are unclear. Patients with a strong Type A personality are more stress sensitive and, as a result, may be more susceptible to diseases triggered by chronic stress $(30,31)$. We therefore hypothesized that the augmented Bortner Type A score observed in

Table 4 Comparison of type 1 diabetic patients and patients with an auto-immune thyroid disease in terms of age, sex ratio, Bortner score, anxiety and depression.

\begin{tabular}{|c|c|c|c|}
\hline & $\begin{array}{c}\text { Type } 1 \\
\text { diabetes }\end{array}$ & $\begin{array}{l}\text { Auto-immune } \\
\text { thyroid diseases }\end{array}$ & $\boldsymbol{P}$ \\
\hline Age (years) & $39.9 \pm 14.3$ & $48.9 \pm 14.8$ & $<0.0001$ \\
\hline Sex ratio (M/W) & $91 / 97$ & $8 / 77$ & $<0.0001$ \\
\hline Bortner (0 to 336) & $188 \pm 34$ & $189 \pm 33$ & NS \\
\hline Anxiety (0 to 21 ) & $9.8 \pm 4.3$ & $10.7 \pm 4.3$ & NS \\
\hline Depression (0 to 21 ) & $5.02 \pm 3.46$ & $5.0 \pm 3.4$ & NS \\
\hline
\end{tabular}

Data are expressed as mean \pm S.D.
T1D could be related to the auto-immune nature of the disease. To verify this assumption, we evaluated the Bortner Type A score in patients with treatable autoimmune thyroid diseases. We found that these patients had elevated Type A scores similar to what was observed in T1D. These data indicate that Type A personality is significantly more common in those with auto-immune diseases. Type A personality is defined by a combination of traits including impatience, strong career ambition and competitiveness. It also includes an increased sensitivity to external stress (21).

Indeed, it has been shown that individuals with Type A personality exhibit a greater sympathetic-adrenal response (increased heart rate and plasma norepinephrine levels) in situations of psychological stress (22). Because auto-immune diseases are known to be triggered by stress $(19,31)$, we may hypothesize that individuals with Type A personality are more likely to develop auto-immune disorders. This is suggested by our data and it can be speculated that Type A personality and auto-immunity share certain psychobiological pathways.

To our knowledge, this is the first time that personality traits were investigated for comparative purposes in individuals with T1D and auto-immune thyroiditis. Interestingly, both groups were found to have an elevated

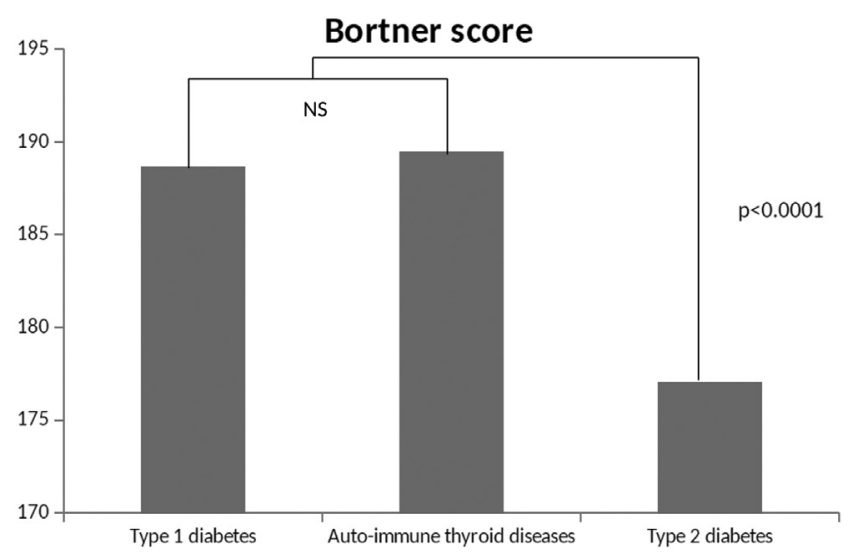

Figure 1

Mean Bortner score in patients with type 1 diabetes $(n=188)$, auto-immune thyroid disease and type 2 diabetes $(n=430)$

This work is licensed under a Creative Commons Attribution-NonCommercial 4.0 International License. ded from Bioscientifica.com at 04/26/2023 10:03:45AM via free access 
Bortner score. This suggests that stress-sensitive Type A people, who are known to be more stress sensitive (15), might be prone to stress-triggered auto-immune disorders such as T1D or immune thyroid diseases (31), as compared to individuals with T2D who are less likely to have a Type A profile. Our data advocate that Type A personality and auto-immunity may share certain psychobiological pathways.

The different personality score between T1D and T2D is an important factor, which could influence self-care coping strategies in diabetes and long-term prognosis. Indeed, Type A personality is characterized by control, organization and assiduousness that may improve selfcare coping strategies in diabetes $(7,8,9,11)$. In addition, Type A personality has been shown to be independently associated with reduced plasma C-reactive protein levels (1) in both T1D and T2D, and with downregulation of c-Fos expression in T1D (32), suggesting that elevated Type A score could be associated with reduced chronic inflammation that may be beneficial. This seems to be confirmed by a prospective study which showed that Type A personality was associated with lower cardiovascular and all-cause mortality in T1D (15).

There are some limitations in our study. First, there were more women in the group with auto-immune thyroid diseases than in the T1D group. This can be explained by the much higher overall prevalence of thyroid diseases in women. However, the Bortner Type A scores were similar for men and women, and the mean Bortner score remained statistically similar for patients with T1D and patients with auto-immune thyroid diseases after adjustment for sex. Thus, the sex ratio difference between the patients groups is not likely to have influenced the results in our study. Similarly, mean age was different for each group but a comparison of Bortner Type A scores after adjustment for age was unchanged, indicating that our results were not biased by difference in age.

Hostility was not measured in this study, as the Bortner scale is not designed for it. It would be interesting to conduct studies to evaluate this parameter in the future.

\section{Conclusion}

Our study found a higher Type A score in patients with T1D when compared with patients with T2D, who showed a mean Type A score comparable to the general population. We show that patients suffering from an auto-immune disease also show a similar augmented Type A score to the one observed in our patients with T1D.
Our data indicate that the augmented Bortner Type A score observed in T1D is likely to be due to the auto-immune origin of the disease. This suggests a possible link between Type A personality and auto-immune diseases via stresstriggering psychobiological pathways. Further studies are needed to find out the precise mechanisms linking Type A profile and auto-immune diseases.

\section{Declaration of interest}

The authors declare that there is no conflict of interest that could be perceived as prejudicing the impartiality of the research reported.

\section{Funding}

This research did not receive any specific grant from any funding agency in the public, commercial or not-for-profit sector.

\section{Acknowledegments}

The authors are grateful for the contribution of all study participants and for the work and dedication of all the endocrinology department staff. The authors thank Véronique Grivet, Cécile Breuiller and Isabelle Simoneau for their expert technical assistance. In addition, the authors gratefully acknowledge Suzanne Rankin for the English revision of the manuscript.

\section{References}

1 Chauvet-Gélinier JC, Trojak B, Lemogne C, Aho-Glélé LS, Brindisi MC, Bouillet B, Ponavoy E, Meille V, Simoneau I, Chahraoui $\mathrm{K}$, et al. Potential influence of Type A personality on plasma C-reactive protein levels in people with diabetes. Diabetes and Metabolism 201642 88-95. (https://doi.org/10.1016/j. diabet.2015.08.001)

2 Šmigelskas K, Žemaitienė N, Julkunen J \& Kauhanen J. Type A behavior pattern is not a predictor of premature mortality. International Journal of Behavioral Medicine 201522 161-169. (https:// doi.org/10.1007/s12529-014-9435-1)

3 Haynes SG \& Feinleib M. Type A behavior and the incidence of coronary heart disease in the Framingham Heart Study. Advances in Cardiology 198229 85-94. (https://doi.org/10.1159/000406201)

4 Franco-Belge GC. Facteurs psychologiques et cardiopathies ischémiques. Annales de Cardiologie and d'Angeiologie 198332 295-302.

5 Bortner RW, Rosenman RH \& Friedman M. Familial similarity in pattern a behavior: fathers and sons. Journal of Chronic Diseases 1970 23 39-43. (https://doi.org/10.1016/0021-9681(70)90107-4)

6 Matthews KA \& Rakaczky CJ. Familial aspects of the type A behavior pattern and physiologic reactivity to stress. In Biological and Psychological Factors in Cardiovascular Disease, pp 228-245. Berlin, Heidelberg, Germany: Springer, 1986. (https://doi.org/10.1007/9783-642-71234-0_14)

7 Hart KE. Association of type A behavior and its components to ways of coping with stress. Journal of Psychosomatic Research 198832 213-219. (https://doi.org/10.1016/0022-3999(88)90057-8)

8 Ramanaiah NV, Sharpe JP \& Byravan A. Type A behavior and the fivefactor model of personality. Psychological Reports 199781 368-370. (https://doi.org/10.2466/pr0.1997.81.2.368)

9 Lemogne C, Turinici M, Panjo H, Ngo C, Canoui-Poitrine F, Chauvet-Gelinier JC, Limosin F, Consoli SM, Goldberg M, Zins M, et al. Personality and breast cancer screening in women of the

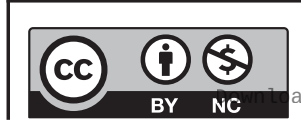

This work is licensed under a Creative Commons Attribution-NonCommercial 4.0 International License. Atrom Bioscientifica com at 04/26/2023 10:03:45AM 
GAZEL cohort study. Cancer Medicine 20187 515-524. (https://doi. org/10.1002/cam4.1268)

10 De Leon M, Carlos F, Powell LH \& Kaplan BH. Change in coronaryprone behaviors in the recurrent coronary prevention project. Psychosomatic Medicine 199153 407-419.

11 Lemogne C, Consoli SM, Panjo H, Nabi H, Goldberg M, Zins M \& Ringa V. Personality and hormone therapy use among postmenopausal women in the GAZEL cohort study. Fertility and Sterility 201298 929-936. (https://doi.org/10.1016/j. fertnstert.2012.06.020)

12 Sensky T \& Petty R. Type A behaviour pattern and glycaemic control in type I diabetes. Psychotherapy and Psychosomatics 198952 96-100. (https://doi.org/10.1159/000288306)

13 Lawson VL, Bundy C \& Harvey JN. The influence of health threat communication and personality traits on personal models of diabetes in newly diagnosed diabetic patients. Diabetic Medicine 2007 24 883-891. (https://doi.org/10.1111/j.1464-5491.2007.02155.x)

14 Giles DE, Strowig SM, Challis P \& Raskin P. Personality traits as predictors of good diabetic control. Journal of Diabetes and its Complications 19926 101-104. (https://doi.org/10.1016/10568727(92)90019-h)

15 Fickley CE, Lloyd CE, Costacou T, Miller RG \& Orchard TJ. Type A behavior and risk of all-cause mortality, CAD, and CAD-related mortality in a type 1 diabetic population: 22 years of follow-up in the Pittsburgh Epidemiology of Diabetes Complications Study. Diabetes Care 201336 2974-2980. (https://doi.org/10.2337/dc13-0266)

16 Robinson N, Stevens LK, Bush LJ \& Fuller JH. Personality characteristics in diabetic patients. Practical Diabetes International 19896 224-227. (https://doi.org/10.1002/pdi.1960060510)

17 Dunn SM \& Turtle JR. The myth of the diabetic personality. Diabetes Care 19814 640-646. (https://doi.org/10.2337/diacare.4.6.640)

18 Čukić I \& Weiss A. Personality correlates of type 1 diabetes in a national representative sample. Psychological Topics 201625 45-58.

19 Stojanovich L \& Marisavljevich D. Stress as a trigger of autoimmune disease. Autoimmunity Reviews 20087 209-213. (https://doi. org/10.1016/j.autrev.2007.11.007)

20 Kåre Berglund L-OP DS. Psychological factors in chronic rheumatic diseases - a review: the case of rheumatoid arthritis, current research and some problems. Scandinavian Journal of Rheumatology 199928 137-144.

21 Shepshelovich D \& Shoenfeld Y. Prediction and prevention of autoimmune diseases: additional aspects of the mosaic of autoimmunity. Lupus 200615 183-190. (https://doi.org/10.1191/096 1203306lu2274rr)

22 Huerta PT, Kowal C, DeGiorgio LA, Volpe BT \& Diamond B. Immunity and behavior: antibodies alter emotion. PNAS 2006103 678-683. (https://doi.org/10.1073/pnas.0510055103)

23 Do Vale S, Martin Martins J, Fagundes MJ \& Do Carmo I. Plasma dehydroepiandrosterone-sulphate is related to personality and stress response. Neuro Endocrinology Letters 201132 442-448.

24 Bortner RW. A short rating scale as a potential measure of pattern A behavior. Journal of Chronic Diseases 196922 87-91. (https://doi. org/10.1016/0021-9681(69)90061-7)

25 Rustin RM, Dramaix M, Kittel F, Degre C, Kornitzer M, Thilly C \& De Backer G. Evaluation of technics used to define type ' $A$ ' pattern, in the Belgian Prevention Project of cardiovascular diseases (author's transl). Revue d'Épidémiologie and de Sante Publique 197624 497-507.

26 Nabi H, Consoli SM, Chastang JF, Chiron M, Lafont S \& Lagarde E. Type A behavior pattern, risky driving behaviors, and serious road traffic accidents: a prospective study of the GAZEL cohort. American Journal of Epidemiology 2005161 864-870. (https://doi.org/10.1093/ aje/kwi110)

27 Zigmond AS \& Snaith RP. The Hospital Anxiety and Depression Scale. Acta Psychiatrica Scandinavica 198367 361-370. (https://doi. org/10.1111/j.1600-0447.1983.tb09716.x)

28 Bucik V \& Brenk K. Internal and external validation of the Bortner Type A behaviour scale. Review of Psychology 19974 25-40.

29 Wang Y, Terao T, Hoaki N, Goto S, Araki Y, Kohno K \& Mizokami Y. Type A behavior pattern: Bortner scale vs. Japanese-original questionnaires. Journal of Affective Disorders 2012142 351-354. (https://doi.org/10.1016/j.jad.2012.03.049)

30 Slavich GM \& Shields GS. Assessing lifetime stress exposure using the stress and adversity inventory for adults (adult STRAIN): an overview and initial validation. Psychosomatic Medicine 201880 17-27. (https://doi.org/10.1097/PSY.0000000000000534)

31 Delévaux I, Chamoux A \& Aumaître O. Stress and auto-immunity. La Revue de Médecine Interne 201334 487-492. (https://doi. org/10.1016/j.revmed.2012.10.366)

32 Chauvet-Gélinier JC, Mosca-Boidron AL, Lemogne C, Ragot S, Forestier N, Callegarin D, Allard C, Rebaï A, Bouillet B, Ponavoy E, et al. Type A competitiveness traits correlate with downregulation of c-Fos expression in patients with type 1 diabetes. Diabetes and Metabolism 201945 582-585. (https://doi.org/10.1016/j. diabet.2018.11.005)

Received in final form 10 February 2020 Accepted 25 February 2020

Accepted Manuscript published online 26 February 2020 https://ec.bioscientifica.com https://doi.org/10.1530/EC-19-0499 (c) 2020 The authors Published by Bioscientifica Ltd

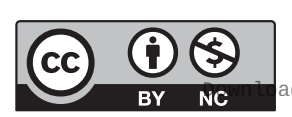

This work is licensed under a Creative Commons Attribution-NonCommercial 4.0 International License. ded from Bioscientifica.com at 04/26/2023 10:03:45AM 